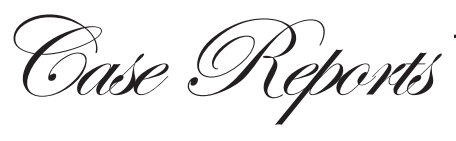

\section{Inserting the Sengstaken-Blakemore tube successfully in a difficult case of uncontrollable esophageal variceal bleeding, using sutures and an endoscope}

\section{Introduction}

Uncontrolled acute esophageal variceal bleeding carries a high mortality rate. When endoscopic therapy fails, a temporising measure such as inserting a Sengstaken-Blakemore (SB) tube secures hemostasis. This allows for patient stabilisation, correction of coagulopathy and arrangement of definitive therapies such as transjugular intrahepatic portal systemic shunting or repeat endoscopy. However, inserting an SB tube blindly carries the potential risk of tube malposition. This may arise due to either the operator's inexperience or a difficult pharyngo-esophageal anatomy. In these cases, inflating the gastric balloon in the esophagus can result in esophageal rupture, mediastinitis and death. ${ }^{1-3}$ Another immediate complication related to the insertion of a SB tube includes inadvertent endotracheal intubation. ${ }^{4-7}$ This may result in tracheal/bronchial perforation or obstruction. At times, insertion of the SB tube can be unsuccessful due to coiling in the posterior pharynx. Failure of insertion of the SB tube and incorrect positioning also results in continued gastrointestinal bleeding.

We describe a case of a difficult SB tube intubation in a patient with uncontrolled esophageal variceal bleeding. The tube was unsuccessfully inserted despite multiple attempts. Using sutures and an endoscope, we successfully inserted the SB tube at the bedside.

\section{Case report}

A 74-year-old male with a medical history of Child B, hepatitis B liver cirrhosis and locally advanced hepatocellular carcinoma presented with hematemesis. After resuscitation and intubation for airway protection, endoscopy was performed in the medical intensive treatment unit.
Gastroscopy revealed grade 3 esophageal varices with active oozing. Endoscopic variceal ligation was performed with hemostasis obtained. However 2 hours later, he had active rebleeding again. Gastroscopy was performed, however the source could not be localised due to rapid pooling of blood in the esophagus. SB tube insertion was attempted, but unfortunately despite multiple attempts, it could not be inserted successfully into the stomach. Initial attempts to inflate the gastric balloon were abandoned as it led to abnormally high gastric balloon pressures. This suggested that the tube was coiling in the esophagus.

Endoscopic assisted insertion of the SB tube (SengstakenBlakemore-Sonde aus weichgummi, Ch.18, Rusch, Germany) using sutures (Perma-hand silk suture size 0; Ethicon, Inc, Somerville, New Jersey, United States) was performed. The tip of the SB tube was anchored with sutures (Figure 1). The suture line was extended by tying 3 sutures with the needle tips cut off, end-to-end, such that the total length was longer than the adult gastroscope (GIF-XQ240, Olympus Optical Co, Ltd., Tokyo, Japan). The suture was pulled through the instrument channel with standard biopsy forceps, such that the SB tube was anchored trailing behind the tip of the endoscope (Figure 2). The other end of the suture was anchored securely at the operator's end of the instrument channel (Figure 3). With the patient well sedated, the endoscope with the SB tube trailing beside was guided into the mouth and down the esophagus, similar to a routine upper

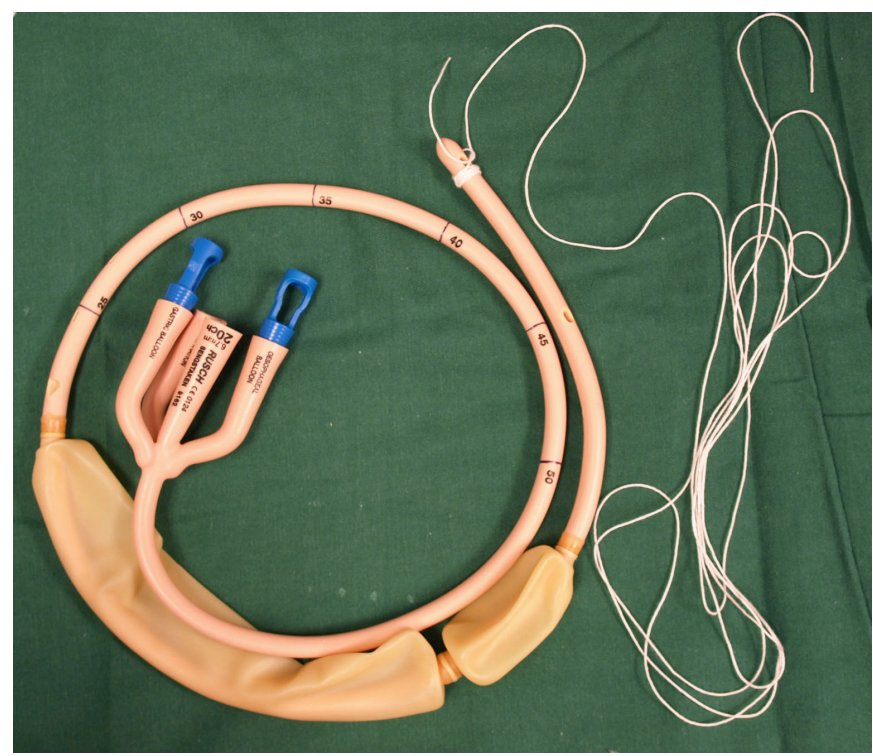

Figure 1: A Minnesota tube (SB tube may be used similarly) is anchored at the tip with a length of thread. Note that either thread or several sutures tied together end to end for sufficient length can be used. 


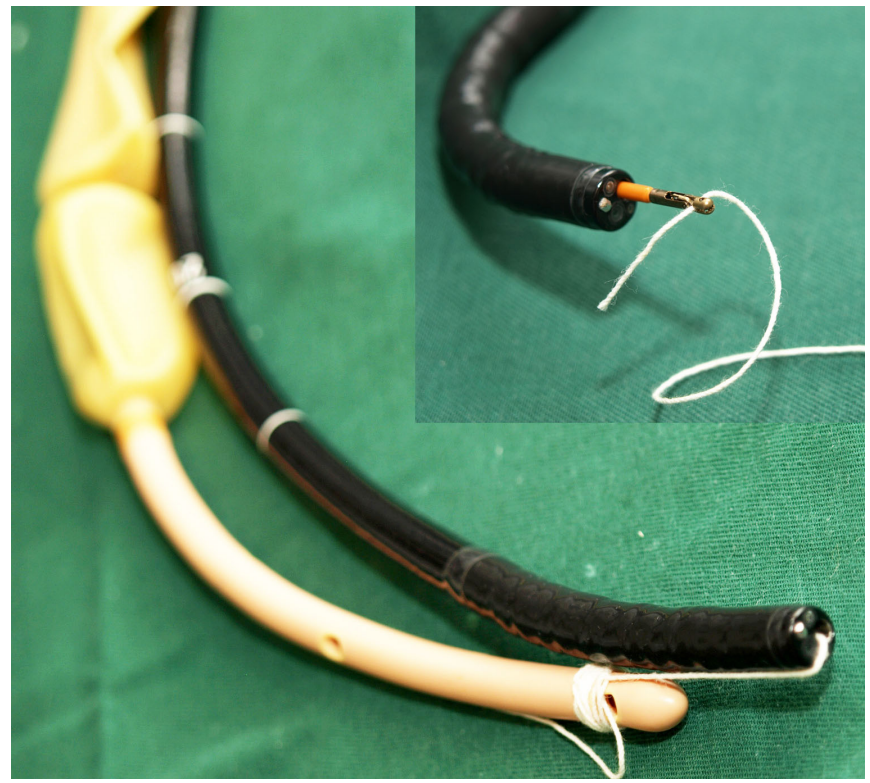

Figure 2: Standard biopsy forceps used to grasp the end of the thread (insert picture). Either thread or sutures may be used. The forceps is withdrawn out of the endoscope, pulling the thread though the instrument channel, anchoring the SB tube by its side.

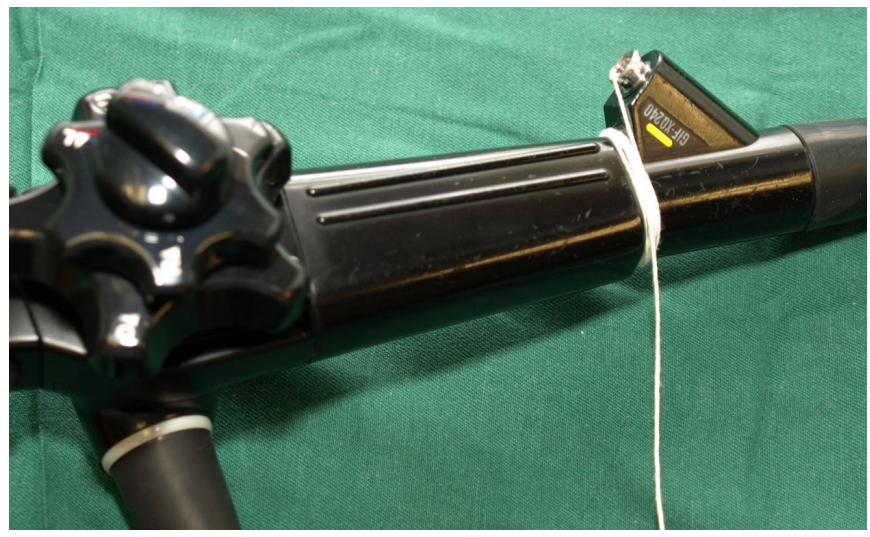

Figure 3: The thread or suture is anchored around the scope by winding it securely around the operator's handle. Note that the instrument channel remains still functional as the thread occupies minimal space.

endoscopy (Figure 4). Within the esophagus the endoscope could be manipulated around the bands of ligated varices, without traumatising the ligated varices and into the antrum.

Within the stomach, the position of the gastric balloon was confirmed with retroflexion of the endoscope and the gastric balloon inflated under direct visualization (Figure 5). The suture was cut at the operator's end, let loose and the endoscope withdrawn leaving the SB tube in-situ. The esophageal balloon of the SB tube was then inflated.

Twenty four hours later the SB tube was removed. The variceal bleeding had ceased and repeat endoscopic variceal ligation was performed immediately with better visualisation of the varices this time due to an absence of active bleeding.

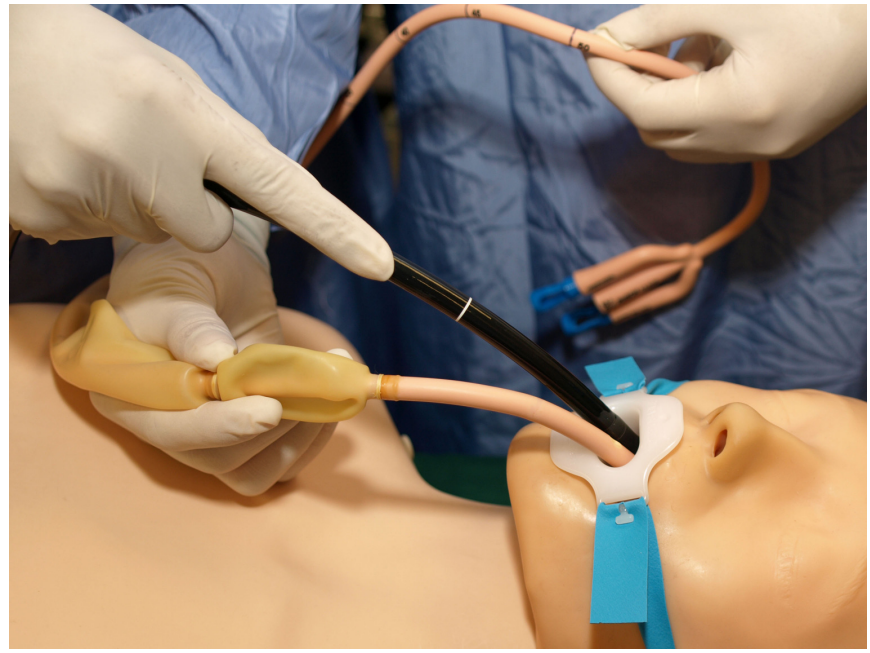

Figure 4: The endoscopist introduces the endoscope into the patient (mannequin used for illustration) in a similar manner to a routine intubation, dragging the SB tube by its side. The assistant guides the SB tube.

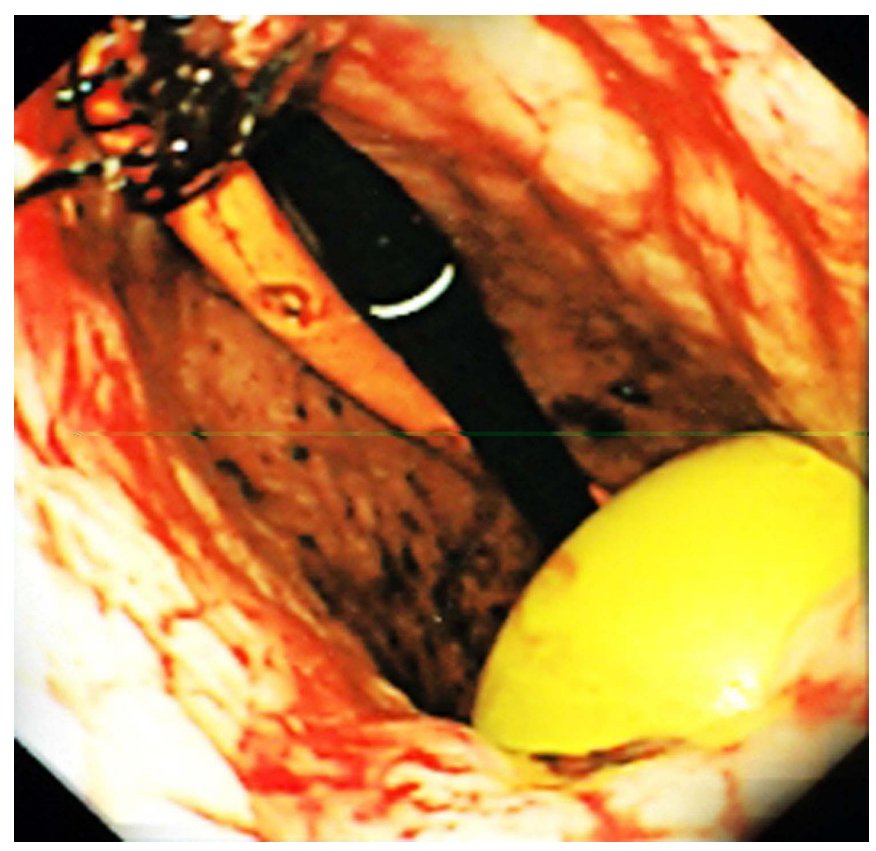

Figure 5: (Actual endoscopic picture) The stomach is lined with fresh blood from an esophageal variceal bleed. The endoscope is introduced deep into the antrum and then retroflexed to confirm that the gastric balloon of the SB tube is within the stomach. The gastric balloon is inflated under direct visualisation. Sutures are seen anchoring the tip of the SB tube.

The patient was eventually discharged from the hospital. At re-look endoscopy 1 month later, there were only grade 1 esophageal varices present.

\section{Discussion}

This is the first reported case of endoscopic assisted insertion of the SB tube using sutures. The merits of this technique are 
that the procedure is easy to perform in difficult cases and sutures are readily available in any hospital.

The adult esophagus is able to accommodate a standard calibre $9.1 \mathrm{~mm}$ adult gastroscope and a SB tube with ease. Once the endoscope with the SB tube has entered the esophagus, angulations, banded varices and diverticular are easily negotiated across or avoided. In a patient with active bleeding varices and clearly failed endoscopic management, securing hemostasis is of utmost importance. The risk of dislodging banded varices with this technique is theoretical and possibly reduced, since the endoscope is inserted under direct visualisation. In comparison, blind insertion of the SB tube in the traditional manner can possibly impinge direct pressure onto the banded varices and may also pose a risk of band dislodgement.

In addition, if a large sliding hiatus hernia is present, the endoscopist will be able to ensure that the gastric balloon is inflated within the stomach and not in the hiatus hernia. One should note that this technique may not be applicable to patients with a narrow esophagus such as a paediatric patient or a patient with an esophageal stricture.

Endoscopic guided insertion of the SB tube by a different method has been described in another case report. ${ }^{8}$ However in that report, an overtube was used to facilitate the insertion of the SB tube past the pharynx followed by an endoscope to push the SB tube down. This is likely to be a semi-blind procedure with the endoscopic view partially obscured. Another option is to use a snare to catch the tip of the SB tube and guide it down the esophagus. Again, with the SB tube and snare in front of the scope, it is likely that endoscopist's view will be compromised. ${ }^{9}$ One benefit of using sutures as opposed to using a snare, is that sutures do not occlude the instrument channel. This allows unobstructed flushing and suctioning of blood when needed which is an important advantage.

Endoscopic insertion of the SB tube allows real-time confirmation of the gastric balloon position, and therefore obviates the risk of intra-esophageal gastric balloon inflation. It does not require guidewires, $\mathrm{X}$-ray or ultrasound confirmation and can be performed at the bedside by any endoscopist. ${ }^{10,11}$ This empowers the endoscopists who have limited experience with SB tube insertions to know with confidence, that it can be successfully performed in difficult cases and that the gastric balloon is placed appropriately.

ERIC WEE ${ }^{1,2}$ LOW HOW CHENG ${ }^{2}$
Gastroenterology, Department of General Medicine, ${ }^{1}$ Khoo Teck Puat Hospital, 90 Yishun Central, Singapore 768828 National University Hospital, ${ }^{2}$ 5 Lower Kent Ridge Road, Singapore 119074 Email:wee.eric.wl@alexandrahealth.com.sg

\section{References}

1 Lin CT, Huang TW, Lee SC, Kuo SM, Hsu KF, Hsu PS, et al. Sengstaken-Blakemore tube related esophageal rupture. Rev Esp Enferm Dig. 2010;102:395-6.

2 Hamm DD, Papp JP. Rupture of esophagus during use of Sengstaken- Blakemore tube. Postgrad Med. 1974;56:199-200.

3 Zeid SS, Young PC, Reeves JT. Rupture of the esophagus after introduction of the Sengstaken-Blakemore tube. Gastroenterology. 1959;36:128-31.

4 Agarwal R, Aggarwal AN, Gupta D. Endobronchial malposition of Sengstaken-Blakemore tube. J Emerg Med. 2008;34:93-4.

5 Chien JY, Yu CJ. Images in clinical medicine. Malposition of a Sengstaken-Blakemore tube. N Engl J Med. 2005;352:e7.

6 Kelly DJ, Walsh F, Ahmed S, Synnott A. Airway obstruction due to a Sengstaken-Blakemore tube. Anesth Analg. 1997;85:219-21.

7 Thomas P, Auge A, Lonjon T, Jean-François, Perrin G, Giudicelli R, Fuentes P. Rupture of the thoracic trachea with a SengstakenBlakemore tube. J Cardiovasc Surg (Torino). 1994;35:351-3.

8 Lin TC, Bilir BM, Powis ME. Endoscopic placement of Sengstaken-Blakemore tube. J Clin Gastroenterol. 2000;31:29-32.

9 Thomson A. Safe and efficacious placement of SengstakenBlakemore tubes. Gastrointest Endosc. 2009;70:401.

10 Kaza CS, Rigas B. Rapid placement of the Sengstaken-Blakemore tube using a guidewire. J Clin Gastroenterol. 2002;34:282.

11 Lock G, Reng M, Messman H, Grüne S, Schölmerich J, Holstege A. Inflation and positioning of the gastric balloon of a SengstakenBlakemore tube under ultrasonographic control. Gastrointest Endosc. 1997;45:538.

\section{Tombstone of surgical clip in common bile duct}

\section{Introduction}

Laparoscopic cholecystectomy has gained popularity over open cholecystectomy due to the benefits of shorter hospital stay, lesser pain and smaller scar. The frequent use of surgical clips during laparoscopic cholecystectomy occasionally leads to migration of the clip into the bile duct. The migrated clip acts as a nidus for stone formation (cat's eye calculus). ${ }^{1}$ These stones 